\title{
KONGRESSKALENDER
}

\section{März 2017}

01.-03.03.2017, Lippstadt-Eickelborn 32. Eickelborner Fachtagung zu Fragen der Forensischen Psychiatrie

Dr. N. Saimeh

Kontaktadresse:

Michaela.Riepe@wkp-lwl.org

Information: www.lwl-forensik-lippstadt.de

21.-22.03.2017, Gießen

Symposium Forensische Psychiatrie

Dr. R. Müller-Isberner

Kontaktadresse: Gisela.Irle@ vitos-haina.de Information:

http://veranstaltungen.forensik-haina.de

\section{Mai 2017}

26.05.2017, Dresden

23. Dresdner Frühjahrstagung

für Forensische Psychiatrie

Sexualdelinquenz - Täter und Opfer

Dr. Lau, Dr. Lammel, Dr. Sutarski,

Prof. Dr. Bauer

Information: www.forensik-dresden.de

28.-31.05. 2017, Mechelen, Belgien

European Association of Psychology

and Law (EAPL)

$2^{\text {th }}$ Annual Conference

Breaking new grounds in Psychology and Law: Futuristic or imminent?

Prof. Dr. Ray Bull, Prof. Dr. R. Volbert

Information: https://eapl.eu

\section{Juni 2017}

07.-09.06.2017, Zürich, Schweiz

9. Internationales Symposium

Forensische Psychiatrie

World Trade Center

Prof. Endrass, Dr. Rossegger, Prof. Urbaniok

Information: www.forensiktagung.ch

9. Juni 2017, Wien, Arcotel Wimberger

11. Wiener Frühjahrstagung

für Forensische Psychiatrie: Recht

oder Rache - der gesellschaftliche

Auftrag des Maßnahmenvollzugs

für zurechnungsunfähige Straftäter

Österreichische Gesellschaft für Forensische Psychiatrie

Prof. Dr. Th. Stompe, Prof. Dr. H. Schanda

Information: www.ce-management.com
13.06.-15.06.2017, Split, Kroatien 17th Annual IAFMHS Conference Forensic Mental Health Services

Hotel Le Meridien LAV

Information: www.iafmhs.org

16. 06. 2017, Berlin-Dahlem

21. Berliner Junitagung für Forensische Psychiatrie und Psychologie Institut für Forensische Psychiatrie der Charité \& Forensische Psychiatrie Psychologie Berlin e.V.

Information: www.forensik-berlin.de (Veranstaltungen)

19.-21.06. 2017, Stockholm, Schweden The Stockholm Criminology Symposium City Conference Center, Stockholm Information:

www.criminologysymposium.com

\section{August 2017}

07.08.-11.08.2017, München und Kloster Irsee

7th Seminar on Forensic Psychiatry in Europe

for consultants and experienced trainees: Communication and Understanding Forensic Psychiatry in a Multiagency World

Prof. Nedopil, Prof. Pamela Taylor,

The Ghent Group

Information: www.bildungswerk-irsee.de

21.-25. 08. 2017, Toronto, Ontario (Canada)

21st Trienniel Meeting of the

International Association

of Forensic Sciences (IAFS)

Sheraton Center Toronto Hotel

Information: http://iafstoronto2017.com

\section{September 2017}

September 2017, Vilnius, Litauen 15th Conference International Association for the Treatment of Sexual Offenders (IATSO)

Information: www.iatso.org

13.-16.09. 2017, Cardiff, Wales (UK)

European Association of Criminology 17th Annual ESC Conference

Information: http://www.esc-eurocrim.org/
27.-30.09. 2017, Kansas City, Missouri (USA)

Association for the Treatment of Sexual Abusers (ATSA)

36th Annual Research and Treatment Conference

Sheraton Kansas City Hotel at Crown Center Information: www.atsa.com/conference

\section{Oktober 2017}

Oktober 2017, München

32. Herbsttagung für Forensische Psychiatrie

Prof. Dr. K. Schiltz

Abteilung für Forensische Psychiatrie der Universität München

Information: www.forensik-muenchen.de

26.-29. 10. 2017, Denver, Colorado (USA)

American Academy of Psychiatry and the Law

$\mathbf{4 8}^{\text {th }}$ Annual Meeting (with APA)

Hyatt Regency Denver

Information: www.AAPL.org

\section{November 2017}

15.-18.11.2017 Philadelphia, Pennsylvania (USA)

American Society of Criminology Annual Meeting

Crime, Legatimacy and Reform - Fifty Years after the President's Commission Philadelphia Marriott Downtown Information: http://www.asc41.com/ annualmeeting.htm

Ein internationaler kriminologischer Kongresskalender findet sich unter http://www.asc41.com/caw.html Hinweise für den Kongresskalender bitte an: mail@hlkroeber.de 